\title{
Changes in Mandibular Third Molar Angulation in High- anchorage Extraction vs Non-extraction Orthodontic Treatment: A Prospective Study
}

\author{
Swati Singh ${ }^{1}$, Arun K Garg ${ }^{2}$, Deepak K Gupta ${ }^{3}$, Litesh Singla ${ }^{4}$
}

\begin{abstract}
Aim and objective: The study aimed to compare the prospective changes in mandibular third molar angulation in high anchorage cases treated with first premolar extractions vs non-extraction orthodontic treatment.

Materials and methods: The sample consisted of 56 nongrowing patients: Group I had 26 patients with a high anchorage requirement who underwent first premolar extractions and group II had 30 patients who underwent non-extraction treatment. Pretreatment, mid-treatment, and posttreatment panoramic radiographs were obtained for group I and pretreatment and posttreatment for group II. Angle between M2 (second molar)-horizontal reference plane (HRP), M3 (third molar)-HRP, and M2-M3 were measured bilaterally. Data were analyzed using Student $t$ test and ANOVA test ( $p$ value $<0.05$ ).

Results: Statistically significant increase was found between the pretreatment, mid-treatment, and posttreatment values of M2-M3 in group I ( $p$ value $=0.02 \mathrm{R}$ and $p$ value $=0.049 \mathrm{~L}$ ) and between pretreatment to posttreatment values of M2-HRP in group II bilaterally $(p$ value $=0.001$ ). Significant increase was found in the M2-M3 angulations in group II on the right side ( $p$ value $=0.036$ ). M3-HRP decreased in group I without reaching statistical significance. No statistically significant intergroup differences were found between the two groups in relation to M2-HRP, M3-HRP, and M2-M3 angulations.

Conclusion: M2-M3 angulations increased significantly bilaterally in group I and on the right side in group II, indicating worsening of third molar angulation. M3-HRP worsened in group I without reaching statistical significance. Extraction therapy in high anchorage cases does not lead to an improvement in third molar angulation.

Clinical significance: The extraction of first premolars in high anchorage cases does not lead to an improvement in the angulation of mandibular third molars; moreover, the angulation worsened with extraction therapy. Prospective orthodontic patients need to be cautioned against any improvement in mesioangular impaction of mandibular third molars in high anchorage premolar extraction cases.

Keywords: Angulation, Extraction, High anchorage, Mandibular third molar, Prospective cohort study.

The Journal of Contemporary Dental Practice (2020): 10.5005/jp-journals-10024-2899
\end{abstract}

\section{INTRODUCTION}

The developmental position and path of eruption of mandibular third molars are known to be very irregular and variable. The same kind of variability is also witnessed in the formation and timing of calcification of these teeth. ${ }^{1}$ Development of mandibular third molar is known to start between 5 years and 14 years of age. ${ }^{2}$ These teeth generally erupt into the oral cavity at around 18-24 years of age. Often, third molars are impacted or in certain cases are congenitally missing. The impaction rate of the third molars (with mandibular teeth more than the maxillary) is highest in the oral cavity with a range from 9.5 to $39 \%,{ }^{3}$ with mesioangular impactions being most common.

A variety of biological factors have been known to contribute to mandibular third molar impaction or the lack of it. These factors include excessive interproximal attrition leading to mesial migration of the posterior segment, growth-related increase in mandibular length, direction of the growth of the condyle, variations in the pattern of ramal resorption, greater size of mandibular third molars, delayed development of facial structures, the pattern and eruptive path of mandibular dentition, and the available retromolar space. ${ }^{4}$ During the developmental stages, the third molars continue to change its angulation by undergoing important "preeruptive rotational movements". Failure of these rotational movements leads to tooth impaction. ${ }^{5}$ ${ }^{1-4}$ Department of Orthodontics and Dentofacial Orthopedics, Dr
Harvansh Singh Judge Institute of Dental Sciences and Hospital,
Panjab University, Chandigarh, India

Corresponding Author: Swati Singh, Department of Orthodontics and Dentofacial Orthopedics, Dr Harvansh Singh Judge Institute of Dental Sciences and Hospital, Panjab University, Chandigarh, India, Phone: +918872362240, e-mail: drswatisingh91@yahoo.com

How to cite this article: Singh S, Garg AK, Gupta DK, et al. Changes in Mandibular Third Molar Angulation in High-anchorage Extraction vs Non-extraction Orthodontic Treatment: A Prospective Study. J Contemp Dent Pract 2020;21(10):1182-1188.

Source of support: Nil

Conflict of interest: None

Prior studies have majorly focused on the effect of the mandibular third molars on the dentition. However, limited data exist on the influence exercised by the rest of the dentition on the mandibular third molars. ${ }^{6}$ Literature is divided regarding the influence of premolar extractions on third molar impaction. Some authors firmly believe that premolar extraction improves the prognosis of mandibular third molars erupting into the correct position and reduces the rate of impaction. ${ }^{5,7-9}$ On the other hand, certain authors believe that third molar position is 
independent of an extraction treatment plan ${ }^{4}$; moreover, some studies have found insignificant differences between extraction and non-extraction subjects. 6,10,11 This issue holds importance even today as premolar extractions indeed provide extra space which is helpful in the treatment of severe tooth-arch discrepancy or even in the correction of sagittal dysmorphosis. Chances of an improvement in the mandibular third molar angulation are often considered while deciding in favor of an extraction treatment plan.

As the literature has showed conflicting results regarding the improvement in mandibular third molars following extraction treatment, the evidence regarding an improvement of mandibular third molar angulation is relatively low. This may be due to the retrospective nature of the previous studies. It has been established that anchorage condition and type of biomechanics employed during treatment have a more significant bearing on the angulation of mandibular third molars over the actual extraction of premolars. ${ }^{6}$ Further, the effect of different types of anchorage has not been studied until recently. ${ }^{12}$ Previous studies conducted on adolescent age-groups carried "a risk of overdiagnosis," as third molar eruption is possible in later stages of root development. ${ }^{13}$ Selection of nongrowing patients with a high anchorage requirement was able to provide an unbiased view regarding the exclusive effect of extractions on third molar angulation, as anchorage loss was minimal.

The aim of this study was to evaluate the changes in mandibular third molar angulation in high anchorage cases indicated for first premolar extraction vs those to be treated without extractions.

\section{Materials and Methods}

The total sample size of 56 nongrowing patients was divided into two different groups in this prospective study. Treatment plan was decided after careful analysis of pretreatment records, that is, clinical photographs, lateral cephalograms, panoramic radiographs, and study models, in the presence of experienced orthodontists. Group I consisted of 26 patients who underwent first premolar extractions, and group II had 30 patients who were treated without extractions. The study was approved by the ethical committee of Panjab University, Chandigarh (Ethical approval number: PUIEC/2018/89/A-1/09/01).

All the patients were treated with Gemini MBT 0.022-inch brackets (3M Unitek, Monrovia, Calif). Pretreatment, mid-treatment, and posttreatment panoramic radiographs were taken for patients in group I. Pretreatment and posttreatment radiographs were taken for patients in group II. All the patients enrolled in the study received the patient information sheet and were briefed by the investigator regarding the study.

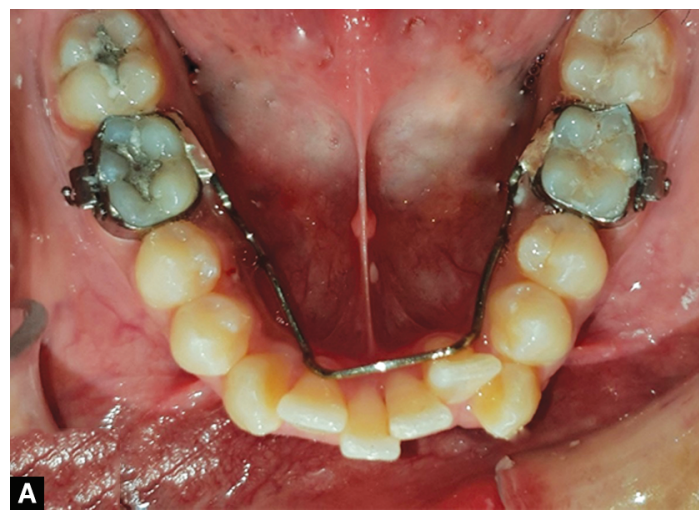

Figs $1 \mathrm{~A}$ and B: Anchorage reinforcement: (A) Lingual arch in hyperdivergent patient; (B) Second molar banding in hypodivergent patient

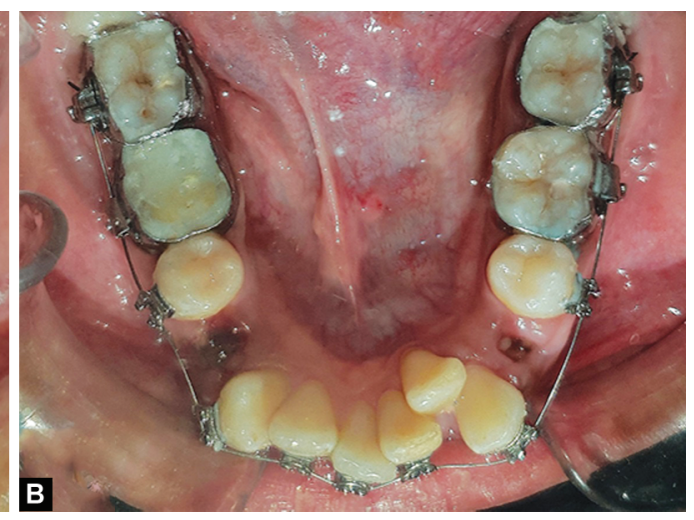

\section{Inclusion Criteria}

- High (critical) anchorage cases (group I).

- Nongrowing patients, 18 years and above or a CVMI of maturational or completion stage.

- Mesioangular impacted mandibular third molars.

- Cases indicated for all first premolar extractions/those to be treated without extractions.

\section{Exclusion Criteria}

- Patients requiring second premolar extraction or mandibular molar protraction.

- Surgical orthodontic (orthognathic) patients.

- Patients having skeletal or craniofacial deformities.

The assessment of the type of mandibular third molar impaction was done with the help of the Winter's classification, ${ }^{14}$ and when the angle between the long axis of the second and third molar was lying between $11^{\circ}$ to $79^{\circ}$, it was termed as mesioangular. ${ }^{15}$

To provide high anchorage in group I, lingual arch was placed prior to leveling and alignment in hyperdivergent patients, and second molar banding was done in normodivergent and hypodivergent patients (Fig. 1). Separate Canine retraction was done to minimize anchorage loss (Fig. 2). In patients with second molar banding, molar stops were made flush with mandibular first molar during separate canine retraction to prevent any loss of anchorage. A mid-treatment radiograph was taken after completion of canine retraction. Anterior retraction was done after making separate units (from canine to molars) in the posterior segment, thus preventing inadvertent anchorage loss (Fig. 3). Mandibular occlusal photographs and panoramic radiographs of a representative patient in group I are presented (Fig. 4).

\section{Procedure for Tracing}

All the panoramic radiographs were traced using matte acetate paper under adequate illumination. The nasal septum and anterior nasal spine were traced and bisected. Following this, a line was drawn perpendicular to the midline bisector passing through the palatal shadow bilaterally. This line served as a constructed plane, which was termed the "horizontal reference plane (HRP)". The outlines of the mandibular third molars and mandibular second molars along with their long axes were traced. The long axis of the second molar and the third molar were defined "as the line traced passing from the mid-occlusal point through the midpoint of the root bifurcation and the midpoint between the mesial and distal root tips" ${ }^{\prime 8}$ (Fig. 5).
- Missing/extracted third mandibular molars. 


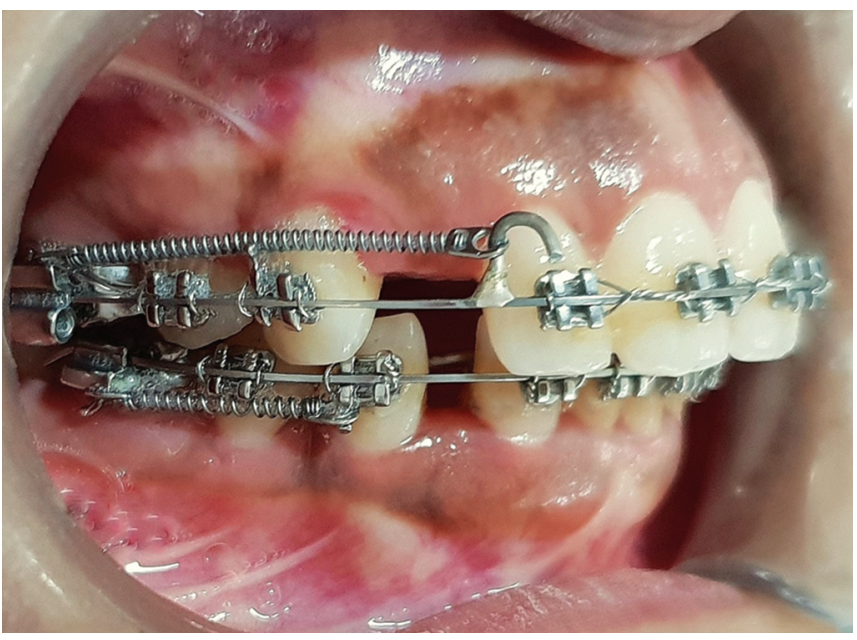

Fig. 2: Separate canine retraction

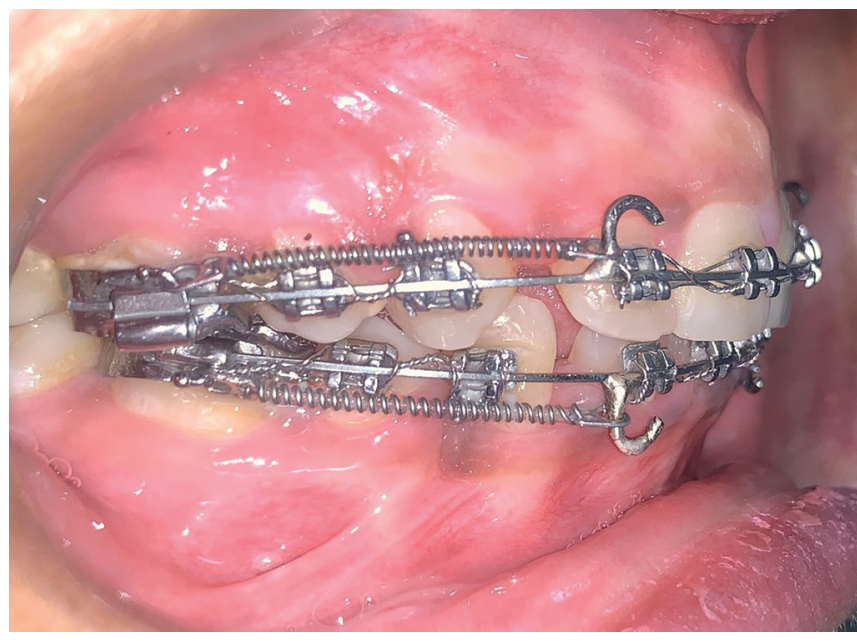

Fig. 3: Anterior retraction
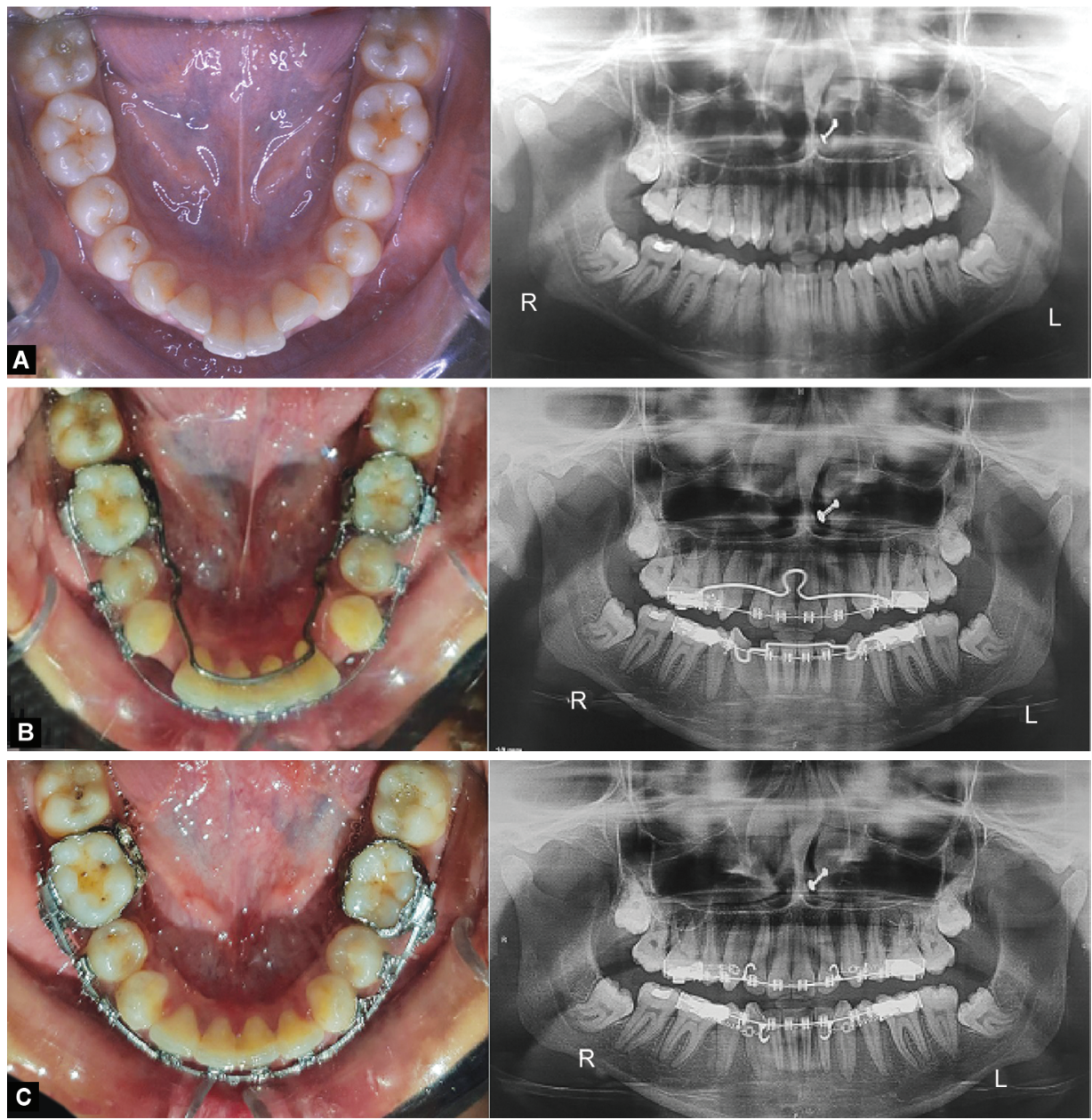

Figs 4A to C: Mandibular occlusal photographs and panoramic radiographs of a representative patient in group I: (A) Pretreatment; (B) Midtreatment (after canine retraction); (C) Posttreatment (after anterior retraction)

\section{Statistical Analysis}

The data obtained in the study were statistically analyzed to test the significance of the findings. Analysis of variance (ANOVA) was done to compare the changes in angular measurements at the pretreatment, mid-treatment, and posttreatment stage in group I. Paired $t$ test was done to compare intragroup pretreatment to posttreatment changes in group II. Unpaired $t$ test was done to compare pretreatment to posttreatment changes in the angulation between group I and group II. The analysis of the interexaminer and intraexaminer reliability was done using intraclass correlation 


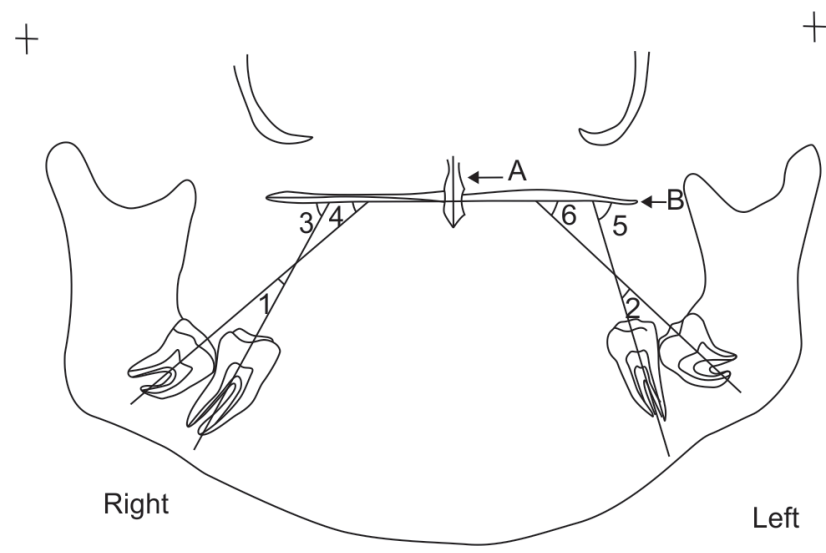

Fig. 5: Diagram showing landmarks and angular measurements: (A) Nasal septum; (B) Horizontal reference plane (HRP); 1, 2 indicate the angulation of M2-M3 (right, left); 3, 5 indicate the angle formed by M2-HRP (right, left); 4, 6 indicate the angle formed by M3-HRP (right, left)

coefficient (ICC). All the statistical analysis were performed on SPSS software for Windows (Ver.22, SPSS. Chicago, III).

\section{Results}

The sample distribution of patients is given in Table 1. Interexaminer and intraexaminer reliability for M2-HRP (Mandibular second molar-HRP), M3-HRP (Mandibular third molar-HRP), and M2-M3 (Mandibular second molar-Mandibular third molar) was checked by retracing the radiograph, and the angular measurements were evaluated again. Interexaminer reliability was checked by one examiner on 15 randomly selected panoramic radiographs, while the intraexaminer was reassessed after a gap of 10 days by the principal investigator. The interexaminer and intraexaminer reliability came out to be highly significant with $p$ value $\leq 0.001$ (Table 2).

ANOVA was done in group I to compare M2-HRP, M3-HRP, and $\mathrm{M} 2-\mathrm{M} 3$ at the pretreatment, mid-treatment, and posttreatment stages. There was no significant difference in M2-HRP on both the right and the left sides in the three stages. M3-HRP decreased by $7.6^{\circ}$ on right and $7.16^{\circ}$ on the left side; however, this change was not statistically significant. The comparison of M2-M3 at the three stages on both the sides revealed statistically significant differences. The mean angle M2-M3 increased significantly ( $p$ value $=0.020$ on the right and $p$ value $=0.049$ on the left side), by $8.33^{\circ}$ on the right side and by $7.92^{\circ}$ on the left side (Table 3).

Paired $t$ test was done to observe pretreatment to posttreatment intragroup differences in group II. The mean angle of M2-HRP increased significantly on the right (by $3.73^{\circ}$ ) and left side (by $4.60^{\circ}$ ) from pretreatment to posttreatment stage in group II ( $p$ value $=$ 0.001). No significant differences were found in the M3-HRP on both the sides in group II. The angulation of M2-M3 increased significantly ( $p$ value $=0.036$ ) by $6.25^{\circ}$ on the right side (Table 4 ).

Intergroup comparisons revealed no significant pretreatment to posttreatment differences in the angulations of the M2-HRP, M3- HRP, and M2-M3 between the two groups (Table 5).

Logistic regression was done to find the relation of gender, growth pattern, and CVMI stage on change in angular measurements of M3-HRP and M2-M3. No significant association of the angular measurements was found in relation to these variables.

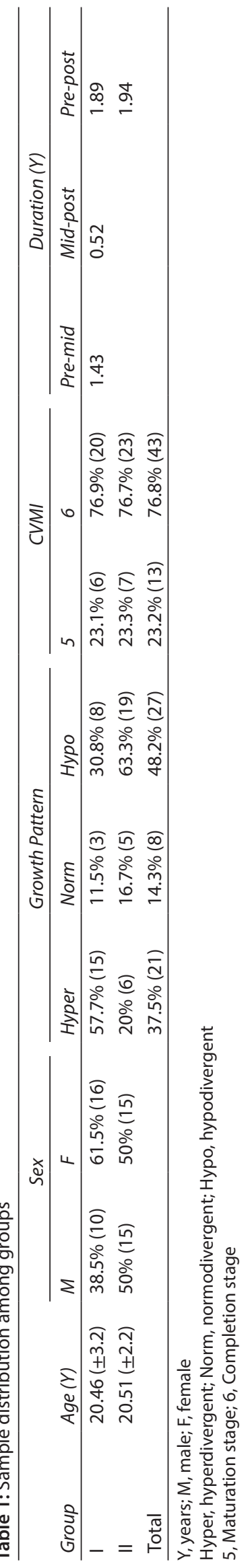




\section{Discussion}

Orthodontics, much like other specialties of science, has its fair share of controversies. The present longitudinal study was designed to address the debate surrounding the changes in mandibular third molar angulations following extraction and non-extraction therapy. A large number of orthodontists firmly believe that extraction of premolars have a favorable influence on mandibular third molars, ${ }^{7-9}$ and ultimately leads to them erupting in good position, while a few others have found insignificant differences between extraction and non-extraction therapy. ${ }^{4,6,11,16}$ On the other hand, it has been suggested that orthodontic treatment that holds back or leads to a distal tipping of mandibular molars may result in unfavorable

Table 2: Intra-examiner and inter-examiner reliability

\begin{tabular}{lcc}
\hline & Inter-examiner & Intra-examiner \\
\hline $\begin{array}{l}\text { Angle from M2-horizontal } \\
\text { reference plane (HRP) } \\
\text { (average measures) }\end{array}$ & 0.883 & 0.928 \\
$\begin{array}{l}\text { Angle from M3-HRP (average } \\
\text { measures) }\end{array}$ & 0.9 & 0.928 \\
$\begin{array}{l}\text { Angle from M2-M3 (average } \\
\text { measures) }\end{array}$ & 0.916 & 0.936 \\
$\begin{array}{l}\text { ICC } \\
p \text { value }\end{array}$ & $0.827-0.936$ & $0.818-0.959$ \\
\hline
\end{tabular}

${ }^{*}$ Highly significant ( $p$ value $\leq 0.001$ ) rotational movements of the mandibular third molars. This would in turn increase the risk of impaction. ${ }^{5}$ However, these studies were retrospective in nature, wherein the treatment methodology could not be efficiently controlled which could have further compromised the results in addition to the selection bias inherently associated with retrospective studies.

In the present study, the mechanics were closely monitored and only nongrowing patients having a high anchorage requirement were included in group I. The palatal plane was chosen as a plane of reference, as it is relatively a more stable landmark than the mandibular and occlusal plane as they undergo remodeling during orthodontic treatment. $^{17}$

In the present study, M2-HRP, M3-HRP, and M2-M3 angulations were measured. In the intragroup comparison, the M3-HRP angle in group I witnessed a mean pre- to posttreatment decrease of $7.6^{\circ}$ on the right and $7.16^{\circ}$ on the left side, although this change was statistically insignificant. The difference in the values of M2-M3 (a mean increase of $8.33^{\circ}$ on the right and $7.92^{\circ}$ on the left side) from pre-, mid-, to posttreatment stage in group I were statistically significant bilaterally indicating a worsening of third molar angulation. The M2-HRP values increased significantly $\left(3.73^{\circ}\right.$ on the right and $4.60^{\circ}$ on the left) in group II indicating a significant amount of uprighting. Pretreatment and posttreatment values in group II for M3-HRP were comparable, but the value of M2-M3 increased significantly on the right side (by $6.25^{\circ}$ ) indicating a greater degree of mesial angulation of the third molars relative to the second molars.

Table 3: Intragroup comparison of angular measurements in group I

\begin{tabular}{|c|c|c|c|c|c|c|}
\hline & \multicolumn{2}{|c|}{ M2-horizontal reference plane (HRP) } & \multicolumn{2}{|c|}{ M3-HRP } & \multicolumn{2}{|c|}{$M 2-M 3$} \\
\hline & Right & Left & Right & Left & Right & Left \\
\hline Pretreatment & $68.08( \pm 6.44)$ & $69.85( \pm 6.83)$ & $55.42( \pm 56.33)$ & $32.33( \pm 13.48)$ & $41.67( \pm 17.03)$ & $37.33( \pm 16.39)$ \\
\hline Mid-treatment & $69.08( \pm 6.84)$ & $70.92( \pm 7.65)$ & $48.83( \pm 59.44)$ & $28.83( \pm 19.96)$ & $50.25( \pm 19.79)$ & $41.58( \pm 22.94)$ \\
\hline Posttreatment & $68.23( \pm 7.04)$ & $69.85( \pm 9.80)$ & $47.75( \pm 60.88)$ & $25.17( \pm 22.28)$ & $50.00( \pm 22.96)$ & $45.25( \pm 24.27)$ \\
\hline$p$ value ( $F$ value) & $0.673(0.792)$ & $0.721(0.653)$ & $0.192(3.30)$ & $0.121(4.217)$ & $0.020 *(7.787)$ & $0.049 *(5.078)$ \\
\hline
\end{tabular}

Angular measurements in degrees \pm SD

*Statistically significant $(p$ value $<0.05$ )

Table 4: Intragroup comparison of angular measurements in group II

\begin{tabular}{|c|c|c|c|c|c|c|}
\hline & \multicolumn{2}{|c|}{ M2-horizontal reference plane (HRP) } & \multicolumn{2}{|c|}{ M3-HRP } & \multicolumn{2}{|c|}{ M2-M3 } \\
\hline & Right & Left & Right & Left & Right & Left \\
\hline Pretreatment & $64.80( \pm 7.12)$ & $65.30( \pm 7.50)$ & $33.91( \pm 15.59)$ & $36.68( \pm 29.16)$ & $32.42( \pm 16.75)$ & $35.12( \pm 16.42)$ \\
\hline Posttreatment & $68.53( \pm 5.56)$ & $69.90( \pm 4.66)$ & $31.39( \pm 25.30)$ & $38.48( \pm 33.56)$ & $38.67( \pm 25.78)$ & $38.08( \pm 24.70)$ \\
\hline Mean difference & -3.73 & -4.60 & 2.52 & -1.80 & -6.25 & -2.96 \\
\hline$p$ value & $0.001^{* *}$ & $0.001^{* *}$ & -0.421 & 0.655 & $0.036^{*}$ & 0.407 \\
\hline
\end{tabular}

Angular measurements in degrees $\pm \mathrm{SD}$

*Statistically significant $(p$ value $<0.05)$

**Highly significant ( $p$ value $\leq 0.001$ )

Table 5: Intergroup comparison of angular measurements

\begin{tabular}{|c|c|c|c|c|c|c|}
\hline & \multicolumn{2}{|c|}{ M2-horizontal reference plane (HRP) } & \multicolumn{2}{|c|}{$M 3-H R P$} & \multicolumn{2}{|c|}{ M2-M3 } \\
\hline & Right & Left & Right & Left & Right & Left \\
\hline Group I & $0.38( \pm 6.51)$ & $1.44( \pm 5.98)$ & $-4.63( \pm 11.12)$ & $-5.57( \pm 15.05)$ & $5.73( \pm 11.14)$ & $6.93( \pm 12.42)$ \\
\hline Group ॥ & $3.73( \pm 4.79)$ & $4.60( \pm 6.03)$ & $-2.52( \pm 14.75)$ & $1.67( \pm 19.12)$ & $6.64( \pm 13.63)$ & $2.96( \pm 17.55)$ \\
\hline Mean difference & -3.36 & -3.16 & -2.10 & -7.24 & -0.91 & 3.97 \\
\hline$p$ value & 0.052 & 0.096 & 0.632 & 0.226 & 0.829 & 0.448 \\
\hline
\end{tabular}

Angular measurements in degrees \pm SD 
In the present study, no statistically significant intergroup difference was seen in the M2-HRP, M3-HRP, and M2-M3 between the two groups. As, M2-M3 values increased in both the groups, the intergroup comparisons revealed no statistically significant worsening of the third molar angulation relative to the second molar, but this result needs to be interpreted with caution as the uprighting of second molars in group II might have confounded this result, especially because in the intragroup comparison, group II did not reveal any considerable change in M3-HRP unlike group I. Moreover, although the M3-HRP angulation did not reveal statistically significant differences, but the third molar angulation worsened to a considerable amount in group I (a decrease of $7.6^{\circ}$ on right and $7.16^{\circ}$ on the left side) unlike group II (a decrease of $2.5^{\circ}$ on right and $-1.8^{\circ}$ on the left side). This result is suggestive of a possibility of an increase in the severity of impaction in high anchorage cases treated with premolar extraction. The results of the present study also throw light on the fact that extraction offers no significant benefit over non-extraction therapy in improving the third molar angulation in nongrowing patients having a high anchorage requirement.

Similar to the present study, Miclotte et al. ${ }^{11}$ reported that no significant differences were found in the mandibular third molar angulation between extraction and non-extraction groups. They also stated that factors such as initial crowding, posttreatment position of incisors, and the mechanics involved along with anchorage could lead to such differences between studies. Staggers et al. ${ }^{6}$ reported insignificant changes in mandibular third found angulation after extraction of premolars and suggested that the third molar angulation is governed by factors other than premolar extractions. However, in the present study, third molar angulations worsened unlike their study where the angles had improved slightly. The reason for this could be attributed to the fact that majority of the patients in their study were adolescents and were undergoing preeruptive uprighting movements. Moreover, as the anchorage requirement was high in the present study, molar protraction was prevented by reinforcing anchorage with the help of lingual arch or second molar banding, and no additional space was available for molar uprighting. In a similar fashion, Artun et al. ${ }^{18}$ studied nongrowing subjects having a mean age of 19 years and concluded that there was no significant difference in third molar angulation between the extraction and non-extraction groups. Further, the suggestions of Artun et al. ${ }^{18}$ were similar to those by Shiller, ${ }^{19}$ Hattab, ${ }^{20}$ and Kruger et al., ${ }^{21}$ in stating that the third molars undergo considerable changes from one direction to another in the later stages of root development. They also suggested that the extent of uprighting achieved during treatment cannot form a predictive model for assessing impaction. Moreover, the mandibular third molars that remained impacted displayed a greater degree of mesial angulation at the end of orthodontic therapy over those that erupted. ${ }^{18}$

Similar to the present study, Azizi et al. ${ }^{12}$ evaluated the effect of premolar extraction on third molar angulation in adolescents having different anchorage requirements. They concluded that minimum and moderate anchorage cases presented with a significant improvement in the third molar angulation. However, the difference in the third molar angulation in high anchorage and non-extraction treatment were insignificant although angulations improved slightly in non-extraction cases. The results of the current study are in close association with the study conducted by Garcia et al. ${ }^{22}$ on an adult sample with a mean age of 19.67 years. The results of their study concluded that the third molars became more vertical in females, while in men, unlike women, the mandibular third molars developed a greater mesial inclination with extraction treatment. In a similar fashion, M3-HRP in the present study also underwent a decrease in group I. Although this result was not statistically significant, it suggested worsening of the mandibular third molar angulation. In the present study, the extraction of first premolars in high anchorage cases was not associated with an improvement in the angulation of mandibular third molars; moreover, the results showed that the angulation worsened with extraction therapy.

Further prospective studies having nongrowing patients with differing anchorage requirement can throw light on the influence of anchorage on mandibular third molar angulation.

\section{Conclusion}

M3-HRP angle decreased in group I, indicating that the third molar angulation worsened with time in the extraction group, without reaching statistical significance. However, angle between M2-M3 increased significantly bilaterally indicating that the third molars became more angulated.

In group II, angle between M2-M3 increased bilaterally, but the change was significant on the right side indicating that the third molar angulation worsened, although, statistically significant uprighting of second molars seen in group II could have confounded the results.

No statistically significant intergroup differences were found between the two groups indicating that extraction therapy in high anchorage cases does not improve the third molar angulation over and above the non-extraction therapy, rather it might deteriorate with time in nongrowing patients.

\section{Clinical Significance}

The extraction of first premolars in high anchorage cases does not lead to an improvement in the angulation of mandibular third molars; moreover, the angulation worsened with extraction therapy. Prospective orthodontic patients can be advised against any improvement in mesioangular impaction of mandibular third molars in premolar extraction cases having a high anchorage requirement.

\section{References}

1. Forsberg CM. Tooth size, spacing, and crowding in relation to eruption or impaction of third molars. Am J Orthod Dentofacial Orthop 1988;94(1):57-62. DOI: 10.1016/0889-5406(88)90451-9.

2. Richardson ME, Dent M. Some aspects of lower third molar eruption. Angle Orthod 1974;44(2):141-145. DOI: 10.1043/00033219(1974)044<0141: SAOLTM>2.0.CO; 2.

3. Richardson M. Changes in lower third molar position in the young adult. Am J Orthod Dentofacial Orthop 1992;102(4):320-327. DOI: 10.1016/0889-5406(92)70047-E.

4. Tarazona B, Paredes V, Llamas JM, et al. Influence of first and second premolar extraction or non-extraction treatments on mandibular third molar angulation and position. A comparative study. Med Oral Patol Oral Cir Bucal 2010;15(5):e760-e766. DOI: 10.4317/medoral.15. e760.

5. Silling G. Development and eruption of the mandibular third molar and its response to orthodontic therapy. Angle Orthod 1973;43(3):271-278. DOI: 10.1043/0003-3219(1973)043<0271: DAEOTM $>2.0 . C O ; 2$.

6. Staggers JA, Germane N, Fortson WM. A comparison of the effects of first premolar extractions on third molar angulation. Angle 
Orthod 1992;62(2):135-138. DOI: 10.1043/0003-3219 ACOTEO>2.0. $\mathrm{CO} ; 2$.

7. Faubion BH. Effect of extraction of premolars on eruption of mandibular third molars. J Am Dent Assoc 1968;76(2):316-320. DOI: 10.14219/jada.archive.1968.0037.

8. Jain S, Valiathan A. Influence of first premolar extraction on mandibular third molar angulation. Angle Orthod 2009;79(6): 1143-1148. DOI: 10.2319/100708-525R.1.

9. Kim TW, Artun J, Behbehani F, et al. Prevalence of third molar impaction in orthodontic patients treated nonextraction and with extraction of 4 premolars. Am J Orthod Dentofacial Orthop 2003;123(2):138-145. DOI: 10.1067/mod.2003.13.

10. Gohilot A, Pradhan T, Keluskar KM. Effects of first premolar extraction on maxillary and mandibular third molar angulation after orthodontic therapy. J Oral Biol Craniofac Res 2012;2(2):97-104. DOI: 10.1016/ j.jobcr.2012.05.004.

11. Miclotte A, Grommen B, de Llano-Pérula MC, et al. The effect of first and second premolar extractions on third molars: a retrospective longitudinal study. J Dent 2017;61:55-66. DOI: 10.1016/ j.jdent.2017.03.007.

12. Azizi F, Shahidi-Zandi V. Effect of different types of dental anchorage following first premolar extraction on mandibular third molar angulation. Int Orthod 2018;16(1):82-90. DOI: 10.1016/j. ortho.2018.01.023.

13. Türköz C, Ulusoy C. Effect of premolar extraction on mandibular third molar impaction in young adults. Angle Orthod 2013;83(4):572-577. DOI: 10.2319/101712-814.1.

14. Winter GB. Principles of exodontia as applied to the impacted mandibular third molar: a complete treatise on the operative technic with clinical diagnoses and radiographic interpretations. St. Louis, Mo: American Medical Book Company; 1926. p. 42.

15. Quek SL, Tay CK, Tay KH, et al. Pattern of third molar impaction in a Singapore Chinese population: a retrospective radiographic survey. Int J Oral Maxillofac Surg 2003;32(5):548-552. DOI: 10.1016/S09015027(03)90413-9.

16. Staggers JA. A comparison of results of second molar and first premolar extraction treatment. Am J Orthod Dentofacial Orthop 1990;98(5):430-436. DOI: 10.1016/S0889-5406(05)81652-X.

17. Nanda RS. Reappraising "Wits". Am J Orthod Dentofacial Orthop 2004;125(2):18A. DOI: 10.1016/j.ajodo.2003.12.012.

18. Artun J, Thalib L, Little RM. Third molar angulation during and after treatment of adolescent orthodontic patients. Eur J Orthod 2005;27(6):590-596. DOI: 10.1093/ejo/cji049.

19. Shiller WR. Positional changes in mesio-angular impacted mandibular third molars during a year. J Am Dent Assoc 1979;99(3):460-464. DOI: 10.14219/jada.archive.1979.0295.

20. Hattab FN. Positional changes and eruption of impacted mandibular third molars in young adults. A radiographic 4-year follow-up study. Oral Surg Oral Med Oral Pathol Oral Radiol Endod 1997;84(6):604-608. DOI: 10.1016/s1079-2104(97)90359-0.

21. Kruger E, Thomson WM, Konthasinghe P. Third molar outcomes from age 18 to 26: findings from a population-based New Zealand longitudinal study. Oral Surg Oral Med Oral Pathol Oral Radiol Endod 2001;92(2):150-155. DOI: 10.1067/moe.2001.115461.

22. Mendoza-García LV, Vaillard-Jiménez E, García-Rocha A, et al. Effect of orthodontic treatment involving first premolar extractions on mandibular third molar angulation and retromolar space. J Clin Exp Dent 2017;9(3):e333-e337. DOI: 10.4317/jced.53434. 